\title{
On the 100th Birthday of Sergei Nikolaevich Chernikov
}

\author{
I. I. Eremin ${ }^{\dagger}$ and A. A. Makhnev ${ }^{1}$ \\ Received May 23, 2012
}

\begin{abstract}
Chernikov's brief biography and information on the International Conference "Algebra and Linear Optimization" (Yekaterinburg, May 14-19, 2012) dedicated to his 100th birthday are presented.
\end{abstract}

DOI: $10.1134 / \mathrm{S} 0081543813090010$

May 11, 2012, was the 100th birthday of prominent Soviet algebraist Corresponding Member of the Academy of Sciences of the Ukrainian Soviet Socialist Republic Sergei Nikolaevich Chernikov. Chernikov was born in Zagorsk (Sergiev Posad), Moscow oblast. His father Nikolai Nikolaevich was a priest, and his mother Anna Alekseevna was a housewife. After finishing secondary school, Chernikov worked as a general laborer. Then, he was trained as a driver and a bookkeeper but found a place as an accountant. Later, he taught mathematics at a workers' school (until November 1931). He registered as an external student at the Department of Physics and Engineering of the Saratov Pedagogical Institute in 1930 and obtained a degree as a teacher of physics and mathematics in 1933. Simultaneously with studying at the institute, Chernikov taught mathematics at an agricultural school in Tatishchev district of Saratov oblast from 1931 to 1932 and physics at Saratov SevenYear Factory School No. 17 during the 1932-1933 academic year. When he graduated from the institute in 1933, People's Commissariat for Heavy Industry of the USSR sent him to Sverdlovsk to teach mathematics at technical colleges of this commissariat. He was an assistant lecturer at the Chair of Mathematics of the Ural Institute of Physics and Mechanics for one academic year. From September 1934, Chernikov worked as an assistant lecturer at the Chair of Mathematics of the Ural Industrial Institute. In January 1939, he finished his studies as an external postgraduate student of the Chair of Algebra of Moscow State University and defended his candidate's dissertation. In the same year, he achieved the academic title of associate professor and was appointed Head of the Chair of Mathematics of the Ural Industrial (later, Polytechnical) Institute in September. He held this position until February 1946. In 1940, Chernikov defended his doctoral dissertation "Locally Soluble Groups" at Moscow State University. In January 1941, he was conferred the academic title of Professor of the Chair of Higher Mathematics. In 1945, by order of the Ministry of Higher Education, he was transferred to Ural State University. From September 1945 to September 1951, Chernikov worked as Professor and first Head of the Chair of Mathematical Analysis at Gor'kii Ural State University. From February 1947 to 1950, he also was Dean of the Department of Physics and Mathematics of the same university.

\footnotetext{
${ }^{\dagger}$ Deceased.

${ }^{1}$ Institute of Mathematics and Mechanics, Ural Branch of the Russian Academy of Sciences, ul. S. Kovalevskoi 16, Yekaterinburg, 620990 Russia; Institute of Radioelectronics and Informational Technologies, Ural Federal University, ul. Mira 19, Yekaterinburg, 620002 Russia

email: makhnev@imm.uran.ru
} 
From September 1951 to May 1961, Chernikov headed the Chair of Higher Algebra and Geometry of Molotov (Perm) State University. In addition, he headed the Chair of Mathematics and Theoretical Mechanics of the Perm Agricultural Institute from September 1955 to 1961. Sergei Nikolaevich earned the sincere respect of the academic staff as a brilliant scientist and talented educator. In particular, Perm University's rector V.F. Tiunov let him leave his post only after Chernikov found a worthy substitute in the person of known algebraist Associate Professor P.I. Trofimov from Tomsk.

In 1961, Director of the Sverdlovsk Division of the Steklov Mathematical Institute of the USSR Academy of Sciences (now, the Institute of Mathematics and Mechanics of the Ural Branch, Russian Academy of Sciences) Professor S.B. Stechkin invited Chernikov back to Sverdlovsk. Sergei Nikolaevich became Head of the newly-created Department of Algebra, where he continued his studies in group theory. At that period, he also paid much attention to another area of research the theory of linear inequalities. Simultaneously with the creation of the Department of Algebra, the Laboratory of Linear Programming was organized under Chernikov's close supervision. His pupil Candidate of Science (now, Academician of the RAS) Eremin, one of the authors of the present paper, became Head of the laboratory.

Chernikov's influence on the development of algebra and mathematical programming, in particular, in the Urals, was marked by the publication of quite a number of brilliant papers in these areas and by the creation of productive scientific schools known both in Russia and abroad.

In 1965, Chernikov's pupil, the founder of the Institute of Cybernetics of the National Academy of Sciences of Ukraine, V.M. Glushkov invited him to Kiev, where Chernikov headed the Department of Algebra at the Institute of Mathematics of the National Academy of Sciences of Ukraine to the end of his life; simultaneously, from 1965, he taught at the Kiev Pedagogical Institute.

After Chernikov left for Kiev, investigations in algebra and linear optimization in Sverdlovsk were continued by the Departments of Algebra and Mathematical Programming headed by Al'bert Ivanovich Starostin and Ivan Ivanovich Eremin.

Chernikov is one of the acknowledged founders of the modern algebraic theory of linear inequalities. During the Great Patriotic War in 1941-1945, Chernikov solved important applied problems, which resulted in the creation of a deep and comprehensive theory of linear inequalities. The theory was widely applied in the theory and methods of optimization, mathematical economics, and pattern recognition. We should mention here the scientific school on the theory and methods of mathematical programming and pattern recognition founded later by Chernikov's pupils Academician Eremin and Professor Vl.D. Mazurov. The Ural School of Optimization and Pattern Recognition differs from other scientific centers by its algebraic approach to the development of mathematical constructions and proof of the results that are mainly based on the theory of linear inequalities built by Chernikov.

Chernikov's achievements include not only the development of a large new chapter of general group theory but also the upbringing of many young scientists and the creation of group-theory schools in Sverdlovsk, Perm, and Kiev. Among his pupils, there are Academician of the USSR Academy of Sciences Glushkov, Academician of the RAS Eremin, Corresponding Member of the USSR Academy of Sciences M.I. Kargapolov, about twenty doctors of science, and more than forty candidates of science.

In 1967, Chernikov was elected Corresponding Member of the Academy of Sciences of Ukraine. He was awarded the Order of Friendship of Peoples (1982), Medal "For Valiant Labor in the Great Patriotic War 1941-1945" (1946), and other medals. 


\section{CHERNIKOV'S SELECTED PAPERS}

\section{8}

"Extension of one Frobenius theorem to infinite groups," Mat. Sb. 3(45), 413-416 (1938).

\section{0}

"Infinite locally soluble groups," Mat. Sb. 7(49), 35-64 (1940).

\section{3}

"On the theory of locally soluble groups," Mat. Sb. 13(55), 317-333 (1943).

\section{4}

"A generalization of the Kronecker-Capelli theorem on a system of linear equations," Mat. Sb. 15(57), 437-448 (1944).

\section{5}

"On the theory of infinite p-groups," Dokl. Akad. Nauk SSSR (N.S.) 50, 71-74 (1945).

\section{6}

"Complete groups with increasing central series," Mat. Sb. 18(60), 397-422 (1946).

\section{7}

"Soluble and nilpotent groups," Uspekhi Mat. Nauk (N.S.) 2(3), 18-59 (1947) [joint with A.G. Kurosh].

\section{0}

"On the minimality condition for abelian subgroups," Dokl. Akad. Nauk SSSR (N.S.) 75, 345-347 (1950).

1951

"On locally soluble groups satisfying the minimality condition for subgroups," Mat. Sb. 28(70), 119-129 (1951).

\section{3}

"Systems of linear inequalities," Uspekhi Mat. Nauk 8(2), 7-73 (1953).

1958

"On layer-finite groups," Mat. Sb. 45(87), 415-416 (1958).

1963

"Contraction of finite systems of linear inequalities," Dokl. Akad. Nauk SSSR 152(5), 1075-1078 (1963).

\section{4}

"Infinite groups with certain prescribed properties of systems of their infinite subgroups," Dokl. Akad. Nauk SSSR 159(4), 759-760 (1964). 
1965

"Polyhedrally closed systems of linear inequalities," Dokl. Akad. Nauk SSSR 161(1), 55-58 (1965).

1968

Linear Inequalities (Nauka, Moscow, 1968); German transl. Lineare Ungleichungen (V.E.B. Deutscher Verlag der Wissenschaften, Berlin, 1971).

\section{9}

"Infinite nonabelian groups with a minimality condition for noninvariant subgroups," Mat. Zametki 6, 11-18 (1969).

1971

"Otto Yul'evich Shmidt (On his eightieth birthday), Ukr. Mat. Zh. 23(5), 581-585 (1971).

\section{2}

"Generalized supersoluble groups with systems of complemented abelian subgroups," Dokl. Akad. Nauk SSSR 205(6), 1306-1309 (1972).

1976

"Some forms of infinite groups with a given system of complemented infinite abelian subgroups," Algebra i Logika 15, 660-683 (1976).

1980

Groups with Prescribed Properties of a Systems of Subgroups (Nauka, Moscow, 1980).

The International Conference "Algebra and Linear Optimization" gathered leading specialists in the area of group theory and its applications as well in the area of mathematical programming and the theory of linear inequalities.

Academician of the RAS V.I. Berdyshev (Director of the Institute of Mathematics and Mechanics of the Ural Branch, RAS), Corresponding Member of the RAS A.A. Makhnev (Co-Chair of the Organizing Committee), and Doctor of Physics and Mathematics Vl.D. Mazurov (representing the Section of Linear Optimization) spoke at the opening of the conference on May 14, 2012.

Twenty plenary talks were given: "The Dual Jordan-Gauss Method" by N.N. Astaf'ev, "On Extensions of Strongly Regular Graphs with Eigenvalue 2" by I.N. Belousov and A.A. Makhnev, "Points of Polyhedra That Are Closest to the Origin" by V.I. Zorkal'tsev, "Factorizations of Groups and Related Issues" by L.S. Kazarin, "Recognition of Finite Groups by Their Prime Graphs" by A.S. Kondrat'ev, "Structural Issues and Automorphisms of Algebras and Groups of Lie Type" by V.M. Levchuk, "Periodic Groups with Additional Conditions of Finiteness" by V.D. Mazurov, "Theory of Linear Inequalities in Chernikov's Papers" by Vl.D. Mazurov, "Edge-Symmetrical Strongly Regular Graphs with At Most 100 Vertices" by A.A. Makhnev and M.S. Nirova, "Newton Methods in the Analysis of Improper Problems of Convex Programming of the First Kind" by L.D. Popov, "The 0-1 Law for Groups and Graphs" by V.N. Remeslennikov, "On Regularization Methods for Improper Problems of Convex Programming" by V.D. Skarin, "Groups with Systems of Frobenius Subgroups" by A.I. Sozutov, "Symmetrical Extensions of Graphs and Their Applications" by V.I. Trofimov, "Computational Complexity and Approximability for a 
Series of Geometric Covering Problems" by M.Yu. Khachai, "Stone Compact Sets in Abstract Attainability Problems with Constraints of Asymptotic Nature" by A.G. Chentsov, "Groups with Minimality Conditions" by N.S. Chernikov, "Semigroups with Finiteness Conditions and Chernikov Groups" by L.N. Shevrin, "Triangulations of Polyhedral Cones and the Realization of Their $f$ Vectors" by V.N. Shevchenko, and "On Groups of Burnside Type" by A.K. Shlepkin.

Twenty-four talks were given at the Section of Algebra, and thirteen talks were given at the Section of Linear Optimization.

One of the strongest results was D.V Lytkina and V.D. Mazurov's progress in the solution of the Burnside problem for groups of period 12. Belousov and Makhnev reported on the completion of a ten-year program of studying graphs in which neighborhoods of vertices are strongly regular with eigenvalue 2. Note that a group of Dutch and Korean mathematicians headed by J. Koolen had intended to investigate this problem. A deep impression on the participants was made by N.S. Chernikov's fundamental talk on groups with minimality conditions. In the area of the theory and methods of optimization, we should first of all note Chentsov's talk on the StoneČech compactification, in which he described a new, topological, approach to the problem of correction of optimization problems with inconsistent constraint systems. Let us also mention Shevchenko and Khachai's remarkable talks in the area of combinatorial optimization and the theory of computational complexity.

Chernikov's memorial evening, held on May 16, brought many new impressions. Participants of the conference paid tribute to the memory of the outstanding scientist and spoke warmly about his personal qualities and diverse interests. Chernikov's pupil Vl.D. Mazurov recollected how Sergei Nikolaevich had supervised his post-graduate students, including Vladimir Dmitrievich and A.N. Fomin. Chernikov's scientific 'grandson' Remeslennikov expressed his regret that he had had little opportunity to communicate with Sergei Nikolaevich during the preparation of his graduation thesis. Chernikov's daughter Natal'ya Sergeevna told about Sergei Nikolaevich's family life during his first 'Sverdlovsk' period, long walks with him (from Ural Polytechnical University's Second Professors' House to the grocery store on Vainera Street), and the complicated relations with him during the 'Perm' period of his life. Chernikov's son Nikolai Sergeevich gave additional details to Natal'ya Sergeevna's story, focusing attention on the reasons for his father's move from Sverdlovsk to Perm. Shevrin spoke about his meetings with Sergei Nikolaevich. Levchuk, Makhnev, Sozutov, and other participants also shared their recollections.

Participants of the conference will long remember the excursion to Ganina Yama and the sightseeing tour of Yekaterinburg.

The closing of the conference was held on Friday, May 18. By tradition, Makhnev informed the participants about the conferences planned for 2013: the 15th All-Russia Conference "Mathematical Programming and Applications" dedicated to Eremin's 80th birthday (Yekaterinburg, FebruaryMarch), the International Conference dedicated to V.D. Mazurov's 70th birthday (Novosibirsk, July), and the Conference on the Theory of Groups and Graphs dedicated to Makhnev's 60th birthday (Yekaterinburg, May). Participants of the conference thanked the organizing committee for the great work on organizing and holding the event. After the closing, a photograph of the participants was taken and a blitz chess tournament was held, in which the winners were Zenkov, Belonogov, and Makhnev.

Translated by E. Vasil'eva 